\title{
Interactive comment on "An Analysis of Conflict and Cooperation Dynamics over Water Events in the Lancang-Mekong River Basin" by Jing Wei et al.
}

\section{Anonymous Referee \#1}

Received and published: 1 October 2020

This paper presents an analysis of conflict and cooperation sentiments in the LancangMekong river basin countries, for which news media articles were analyzed. The general idea and outcomes are relevant and timely. It is also hopeful that once more it is demonstrated that water more often results in cooperation than in "water wars". However, I think that the paper should be improved substantially before it can be considered for publication in HESS. First, it is not clear what the goal of the paper is. I would strongly encourage to make it very clear in the abstract and introduction what the authors aim to achieve with the paper. As a reader, it makes it sometimes difficult to follow. Second, the general structure may be improved. Since the goal is not directly clear, it is also not logical to first present the case study in detail. I would suggest to make sure 
it is clear what research question is being answered, followed by a discussion of the chosen/developed method. Overall, I also think the methods are not presented well. Especially since the chosen methods are perhaps not trivial for the HESS readership, this should be clearly explained earlier in the manuscript. Third, in the introduction a case is made for using socio-hydrology for understanding transboundary river challenges. In the rest of the paper, to link is made to socio-hydrology. I would encourage to make this link in the discussion section. Finally, the manuscript would benefit from a thorough and critical discussion of the methods and results. It is not clear at all what assumptions are made and what uncertainties are introduced in the analysis. In conclusion, the paper has potential, but needs some additional work. Please find below some general comments on all sections, and several specific comments.

\section{General comments}

Introduction The introduction provides a good context for this paper. I do think that the step from water related conflicts and transboundary river basin management to sociohydrology $(\mathrm{SH})$ is a bit sudden. I suggest to dedicate a short section on introducing $\mathrm{SH}$ in this context, and better argue for why $\mathrm{SH}$ is a suitable approach. Second, it is not clear to me what the goal of the paper is. Are you presenting a new method? Are you testing a hypothesis? Are you presenting a new perspective? Please make it very clear in the introduction what you aim to achieve with the paper.

Case study Nice introduction to the basin. I would suggest to rearrange the sections a bit. Perhaps first discuss the history of events (line 145-163), before discussing the stakes and interests of the individual countries (lines 129-144).

Methods The methods should be explained more clearly. The first section of the methods is an overall introduction to "news media" and for the approach the reader is referred to Figure 2 (line 183). Almost eight pages in, it still not clear to me what this paper is about or what method has been developed/used to answer what questions. I suggest to rearrange sections 2 and 3 , and start with a very clear description of the goal

Printer-friendly version

Discussion paper 
of the paper and an overall introduction to the methods. After detailing the components of the method, a description of the case study can be given.

Please provide additional details on how the news article search was conducted. What timeframe was used? What languages of news were considered? What were the generated "results"? Individual newspaper articles or also other media?

As sentiment and topic analysis is not commonly used among the HESS readership, I suggest to maybe illustrate the methods with some examples and even a diagram. This can be in the supplementary materials. For example, show how words and lines are analyzed and what words influence the AFINN score.

Results The results are interesting, but read somewhat bulky. Is it possible to present the results in a more concise way? E.g. focus on the main points.

Discussion and Conclusion I suggest to use some subheadings, especially since you describe "key points". Would be great to have those points clearly visible as subheadings. Can you elaborate on why dam infrastructure and development is associated with negative sentiment? Can you elaborate on how this cooperation and collaboration has been achieved?

I'm missing a critical evaluation of the chosen method. Are there any sources of uncertainty? Are there any assumptions made about the news media used (e.g. "free journalism" vs "state-run"). What can be improved about the method? Can it be directly applied to other river basins?

Specific comments Line 22: Can you elaborate a bit on the method/analysis that was used for this paper?

Line 26: Include the six states.

Line 27: What do you mean with "international countries"?

Discussion paper

Line 43: "world's freshwater" -> incorrect, this should be global river flow. 
Line 53: Given the somewhat outdated references, do you agree that the idea of "water wars" is also outdated?

Line 67-72: Can you elaborate on why a socio-hydrological approach may be a suitable approach?

Line 76: Does the TFDD include water incidents after 1999? If so, to what date? Can you explain what this -7 to +7 scale is based on and what it means?

Line 86: Omit "often".

Line 88: Can you specify what is defined as "war", "conflict" and "cooperation" in this context?

Line 121-124: Please rephrase, this is a very long sentence.

Line 167: Specify "news media".

Line 185: Provide reference to the Lexis-Nexis database.

Line 189: What do you define as riparian states? What is this claim based on?

Line 185-192: Not clear what languages you included in your analysis. Only English articles?

Line 204: Who read all 12,316 articles? The same person or a team?

Line 206: When was an article considered not relevant?

Line 225: Spell out AFINN.

Line 239: What ten topics were selected?

Line 250: English language articles? What about the overall trend in news articles in the same period? E.g. what is the influence of just overall more news?

Printer-friendly version

Line 354: Why is it important?

Discussion paper 
Line 388-389: Unclear sentence ("riparian is over-critical"), please rephrase.

Fig. 1: I suggest to also use a background color for the basin in Cambodia and Vietnam. It now looks like the Lancang-Mekong basin stops at Kratie (Cambodia).

Fig. 2: Barely referred to. Either discuss more explicitly or remove.

Fig. 3: Difficult to distinguish the various countries. Try to make the figure clearer, e.g. put the countries on the right side of the figure.

Fig. 4: Very messy figure. Be consistent with percentages (e.g. Fig. 4A has 0-1 scale, unlike Fig. 4B). Also I suggest to only use once decimal for the percentages in Fig. 4B.

Fig. 5: Is there a reason why the words/topics are not spelled out? It reads very messy. (e.g. "countri"). Also I suggest to make it more clear that the three subfigures are for three specific years. E.g. add it to the figures rather than only in the caption.

Fig. 6: Please improve the quality. Specifically the scale of the bubbles in Fig. 6B is not very clear.

Fig. 7: Can you please use the same scale on the y-axis? That would allow a better comparison. Also you can remove the legend in each sub-plot.

Interactive comment on Hydrol. Earth Syst. Sci. Discuss., https://doi.org/10.5194/hess-2020390, 2020. 